\title{
Feasibility of delivering HPV vaccine to girls aged 10 to 15 years in Uganda
}

\author{
Emmanuel Mugisha ${ }^{1}$, D. Scott LaMontagne ${ }^{1}$, Anne R Katahoire ${ }^{2}$, Daniel Murokora ${ }^{3}$, \\ Edward Kumakech ${ }^{1}$, Rachel Seruyange ${ }^{4}$, Vivien Davis Tsu ${ }^{1}$
}

1. PATH, Seattle, USA and Kampala, Uganda

2. Child Health and Development Centre, Kampala, Uganda

3. Uganda Women's Health Initiative, Kampala, Uganda

4. Ministry of Health, Kampala, Uganda.

\begin{abstract} demonstration project exploring the feasibility of two delivery strategies. and focus group discussions.

DOI: http://dx.doi.org/10.4314/ahs.v15i1.5

\section{Introduction}

Globally, cervical cancer is the third most common cancer in women with more than $85 \%$ of the burden in developing countries. ${ }^{1}$ The majority of cervical cancer mortality occurs in developing countries, where screening and optimal treatment are not adequately available. ${ }^{2}$ In the Kampala area, analysis of the cancer registry estimated age-standardized incidence of cervical cancer at 44.1 per 100,000 females for the period 1995-97. ${ }^{3}$
\end{abstract}

Background: Cervical cancer is a leading cause of mortality among women in Uganda. The availability of the human papillomavirus (HPV) vaccine presents an opportunity to prevent cervical cancer. The Government of Uganda conducted a

Objective: To explore the feasibility of two HPV vaccine delivery strategies: 1) a stand-alone school-based strategy that selected girls based on their enrolment in grade 5 (known as the "grade-based" strategy; and 2) an age-based strategy that delivered the HPV vaccine based on the girls' age (10-year-olds). This strategy combined the delivery of the vaccine with the distribution of deworming medication and vitamin A through an existing Child Days Plus program.

Methods: A qualitative study that explored the feasibility of the two delivery strategies from the perspective of health workers, district leaders, and staff of the Uganda National Expanded Programme on Immunization, utilizing in-depth interviews

Results: Coverage data showed that more girls $(88 \%)$ were vaccinated using the grade-based strategy and completed all three doses compared to those $(73 \%)$ vaccinated using the age-based strategy. Health workers and teachers indicated that determining vaccination eligibility was easier by grade than by age and there were minor disruptions to health services and school programs during vaccinations, as reported by health workers and teachers using the grade-based strategy.

Conclusion: HPV vaccine delivery at schools using grade eligibility was more feasible than selecting girls by age. Lessons learned in Uganda could be relevant for countries considering implementing HPV vaccinations.

Keywords: HPV, vaccination, immunization, cervical cancer, school-based program, adolescent girls

\section{Corresponding author:}

Emmanuel Mugisha

PATH, Seattle, USA and Kampala, Uganda

PO Box 24578, Kampala, Uganda

Email: emugisha@path.org

Phone: (256-414) 335500

Fax: (256-414) 335588
Two vaccines to prevent human papillomavirus (HPV) infection, the cause of cervical cancer, are now approved for use in over 120 countries, creating an opportunity to greatly enhance prevention of cervical cancer. However, challenges may exist with delivery of the vaccine to girls aged 9 to 13 years, the recommended population for HPV vaccinations by the World Health Organization $(\mathrm{WHO})^{4}$, as routine immunizations in most national programs target children younger than 5 years of age. ${ }^{5,6}$

Delivering HPV vaccine to young adolescent girls may therefore require a different kind of health programming., ${ }^{4,7}$ The Uganda Ministry of Health, in collaboration with PATH, an international non-profit organization, carried out an HPV vaccination demonstration project using existing human resources, structures, and systems of the Expanded Programme on Immunization (EPI) from 2008 to 2009 to explore the feasibil- 
ity of two HPV vaccine delivery strategies: 1) a stand- based strategy. In the grade-based strategy, all P.5 classes alone school-based strategy that selected girls based on were included, regardless of whether classes were held their enrolment in primary grade 5 (P.5) (known as the in the morning time or afternoon. Both strategies in"grade-based" strategy); and 2) a strategy that com- corporated mechanisms for reaching out-of-school girls bined delivery of HPV vaccine for girls selected based who were aged 10 years, including referral to neighboron their age (10-year-olds) with the distribution of de- ing health units or through regularly scheduled routine worming medication and vitamin A through the exist- outreach sessions for other vaccines. The bivalent HPV ing Child Days Plus (CDP) program (called the "age- vaccine was used (Cervarix ${ }^{\circledR}$, GlaxoSmithKline, Unitbased" strategy). ${ }^{8}$

The entire HPV vaccine demonstration project evaluated the coverage achieved by the strategies, acceptability of HPV vaccine by parents and eligible girls, feasibility of delivery within the existing EPI program, and the incremental economic and financial costs of implementation. The findings from the coverage, acceptability, and cost evaluations have been published elsewhere. ${ }^{8,9,10,1}$ The objective of the feasibility study in this paper was to assess whether school-based delivery selecting girls by grade or community based-delivery through the CDP program selecting girls by age was the best strategy for HPV vaccine delivery in the Ugandan context.

\section{Methods}

HPV vaccination program

Results from previously conducted formative research in Uganda suggested that two delivery strategies in school may be appropriate in the Ugandan context. ${ }^{12,1}$ High school enrolment, due to the institutionalization of Universal Primary Education, suggested that schools could be a viable venue for vaccinations. The research also noted that the twice-yearly CDP program was delivering vitamin $\mathrm{A}$, albendazole for de-worming, and catch-up vaccinations in communities every six months, and often used schools as a venue for distribution. ${ }^{12}$ The CDP program already had funding for health workers to travel outside of facilities to locations in their community. In consultation with the Ministry of Health $(\mathrm{MOH})$ and the Uganda National Expanded Program on Immunizations (UNEPI), the districts of Ibanda (about 300 kilometers southwest of Kampala) and Nakasongola (about 120 kilometers due north of Kampala) were selected, based on size of eligible target population, regional balance, political stability, accessibility, routine immunization performance, and willingness of district leaders to participate.

In the Ibanda District, a grade-based strategy was implemented, and Nakasongola implemented an age-

and high) derived from the district vaccination reports. District leaders and program managers at the national level were also interviewed based on their involvement in the vaccinations.

\section{Data collection process}

Data was collected using key informant interviews and focus group discussions (FGDs). The FGDs mainly served the purpose of validating the information from the key informant interviews (KIIs). Various documents such as registers for HPV vaccinations, HPV training manuals, microplans, training reports and support supervision reports were also reviewed and the information used to supplement the data collected. The interviews and FGDs conducted with the health workers and teachers explored their perspectives on the feasibility of the different vaccine delivery strategies.

Table 1: Study population, data collection method and sample size

\begin{tabular}{ll}
\hline Study population & Data collection method and sample size \\
\hline Health workers & 133 structured interviews and 6 FGDs \\
Teachers & 253 structured interviews and 7 FGDs \\
$\begin{array}{l}\text { National health officials } \\
\text { (UNEPI) }\end{array}$ & 3 key informant interviews \\
$\begin{array}{l}\text { Number of health } \\
\text { facilities and schools in } \\
\text { the sampling frame }\end{array}$ & 31 health facilities and 52 schools \\
Records review & Registers for HPV vaccinations, HPV training \\
& $\begin{array}{l}\text { manuals, microplans, training reports, support } \\
\text { supervision reports }\end{array}$ \\
\hline
\end{tabular}

\section{Data management and analysi} views and FGDs were transcribed in the local languages prior to being translated into English. The translated KIIs and FGDs were then analyzed by the research team using thematic analysis. Since the questions in the guides were organized around broad themes, the first level of analysis was to identify sub-themes emerging within each of the broader themes; these formed the
The qualitative data generated from the in-depth inter-

basis for the construction of a code book. Using Atlas.ti (version 5.0) software (Berlin, Germany), text was coded to the identified themes and sub-themes for further analysis. Representative quotes were used to illustrate key findings of the thematic analyses.

Data triangulation for different phases of data colleclected based on their involvement in the vaccination exercise from 31 health facilities and 52 schools that were
sampled based on vaccination coverage achieved (low 
tion of views and experiences of all study populations. This study was approved by the PATH Research Ethics Committee (USA), Makerere University Institutional Review Board (Uganda), and the Uganda National Council for Science and Technology. All participants provided verbal consent.

Results

Vaccine delivery through the two alternative strategies The reported coverage using administrative data from the two project districts ranged from $85.1 \%$ to $105 \%$ (Table 2) and demonstrates the feasibility of two different delivery strategies for HPV vaccine to young adolescent girls.

\section{Table 2: HPV vaccine uptake from UNEPI routine administrative data, Ibanda and}

$$
\text { Nakasongola Districts, 2008-2009 }
$$

\begin{tabular}{lccc}
\hline Vaccination strategy & $\begin{array}{c}\text { Estimated total } \\
\text { number eligible } \\
\text { girls }\end{array}$ & $\begin{array}{c}\text { Total number (\%) } \\
\text { girls received 1 } \\
\text { dose }\end{array}$ & $\begin{array}{c}\text { Total number (\%) } \\
\text { girls received all } 3 \\
\text { doses }\end{array}$ \\
\hline $\begin{array}{l}\text { Ibanda: Grade-based delivery } \\
\text { strategy (P.5) }\end{array}$ & 3,459 & $3,447(99.6 \%)$ & $3,038(87.8 \%)$ \\
$\begin{array}{l}\text { Nakasongola: Age-based delivery } \\
\text { strategy (Age 10) }\end{array}$ & 2,263 & $3,277(144.8 \%)$ & $2,388(105.5 \%)$ \\
& & & \\
\end{tabular}

There were variations in the coverage achieved by the different strategies with the grade-based strategy achieving higher coverage than the aged-based strategy Interviews and discussions with the health workers and teachers involved in the vaccination exercises in the two districts reveal why there were these variations and their perspectives regarding the two strategies.

\section{Eligibility determination}

The ability to accurately select girls according to the criteria reflected one aspect of feasibility.

Discussions with teachers and health workers revealed that establishing eligibility was easier with the gradebased strategy compared to the age-based strategy. There were 3,459 girls eligible for the grade-based strategy and 2,263 girls eligible for the age-based strategy (Table 2). While health workers and teachers were trained on how to determine eligibility for their respective strategy prior to vaccination, in reality this proved to be more difficult especially for those teachers using the age-based strategy.

Teachers and health workers found it more challenging to accurately determine which girls were 10 years of age across the many grades in school. Determining the eligibility of the girls was easier in the grade-based all in one class all at the same time with no class shifts; achers knew their pupils by name and could easily identify those who were absent and who needed to be reminded to go to the health unit for any missed doses.
Identification of girls for follow-up vaccinations In the age-based delivery strategy, the dropout rate between dose 1 and dose 3 was as high as $27 \%$, while in the grade-based strategy, the dropout rate was only $12 \%$. According to the health workers, the two principa reasons for dropout were absenteeism from schools on the day of vaccination and change of school to schools outside the district. The identification of girls who were absent was much easier in the grade-based strategy since the teachers knew their pupils and could follow them up and remind them to go for the missed doses.

With the aged-based strategy, however, where the girls were scattered across classes, this was more difficul because it involved several class teachers; not all these class teachers were following what was happening with the vaccinations and therefore could not easily identify the girls who were absent and who needed follow-up. A few teachers expressed concern that only one day was given per school for vaccination and that this might have contributed to the dropout rates. There were, however, a few health workers and teachers that made efforts to track the unvaccinated girls and ensure the got vaccinated either from the school through return visits or at a health facility

"The girls who missed vaccination we would go back to their schools and look for them." (Health worker, Ibanda district, grade-based strategy)

In the Nakasongola district, first and second doses of the program's first year were delivered in one school-calendar year while the third dose was delivered in another school-calendar year. According to the health workers some of the dropouts could have been pupils who changed schools after the end of an academic year.

\section{Teacher involvement}

Although vaccinations in schools generally require the cooperation of teachers, delivery of the HPV vaccination in schools goes beyond this, as teachers actively participate in program administration during vaccination sessions at school. In interviews with teachers, the indicated that their involvement in HPV vaccination included registration of eligible girls, follow-up of girls to ensure they got fully vaccinated, reporting of adverse events, and assisting with day-of-vaccination logistics.

Teachers reported that they were able to help the girl understand cancer of the cervix and why they should be vaccinated. They also reported that they sensitized not only the girls but also their fellow teachers, and in some cases they also organized meetings for parents at school. Participation by teachers was considered positive:

"When the health worker came to vaccinate the girls on the first day, seven girls were absent from school... so when they came, I took them to a health facility and she immediately left everything she was doing and vaccinated them." (Teacher in Nakasongola District, age-based strategy). Other challenges in program implementation occurred where teachers were transferred to other schools, teacher absenteeism, or in a few isolated cases, unwillingness of teachers to provide assistance.

\section{Impact on schools}

School disruptions occurred on vaccination days, but less so in the grade-based strategy. The age-based strategy noted challenges with finding girls aged 10 years in multiple classes at each school (the venue for vaccinations). Approximately one-third of teachers interviewed in this study mentioned the process of vaccinations was disruptive to class activities; however, these same teachers overwhelmingly $(>90 \%)$ said the disruption was worth the benefit of vaccinating girls to prevent cervical cancer and all were willing to participate in a similar exercise in the future.

\section{Impact on health centers}

Given that implementation relied on the existing resources and no extra personnel were hired specifically for this program, some impact on health services was observed. According to health workers, over half of the health units did not have the required staff capacity even before implementation of the HPV vaccination activities. Therefore, during actual implementation of HPV vaccination, some staff were temporarily moved or re-deployed from certain settings, such as the outpatient department or antenatal care, to support the provision of HPV vaccination services. Both delivery strategies exerted the same amount of pressure on the already inadequate health workforce.

"The number of patients increased because wheneve she goes for outreach the health unit remained closed and on return finds a backlog of patients...the health unit would be closed for six to eight hours on vacci- 


\section{Cold chain and vaccine handling}

HPV vaccine delivery provided an opportunity to assess deficiencies in the cold chain prior to vaccination and strengthen the system. For example, district managers interviewed noted that two additional refrigerator units were installed by UNEPI and other faulty units repaired. The delivery of HPV vaccine was timed to be concurrent with that of other routine vaccines, resulting in transport efficiencies and program cost savings. Review of temperature records found that temperatures were almost always in range $\left(+2^{\circ} \mathrm{C}-+8^{\circ} \mathrm{C}\right)$. Vaccine wastage was very low: $0.1 \%$ in the Ibanda district and $0.3 \%$ in the Nakasongola district.

\section{Adverse events}

According to records provided by district managers, only six adverse events were reported through the routine adverse events following immunization monitoring program. Pain at the site of injection, feeling faint, eye infection three days after vaccination, and nausea with headache and fever were reported. The three nausea cases occurred among a group of 12 girls who were inadvertently administered dose 3 two weeks after dose 2. None of these events required hospitalization, and no serious adverse event was reported.

\section{Government endorsemen}

The fact that the HPV vaccinations were being implemented by the District Health Office and involved the District Education Office influenced program ac ceptance by health and education personnel. Political and technical leadership at the district level publicly endorsed the program and considered their participation as part of their official duties. In the Nakasongola District, where HPV vaccination was implemented as part of CDP, it was even more clearly a government-endorsed program, as CDP was an ongoing program supported through the $\mathrm{MOH}$

Teachers interviewed mentioned that parents agreed to have their children vaccinated because HPV vaccine was delivered as a government program.

"There were parents who wanted to prevent their children from being vaccinated. The head teacher invited parents to a meeting and explained that this was a gov- ernment program and was being implemented by the District Health Office; after that they agreed." (Teache in the Nakasongola district, age-based strategy)

\section{Discussion}

This qualitative study of HPV vaccine delivery in two districts of Uganda demonstrated the feasibility of two different strategies and illustrated factors that facilitated success and those that presented obstacles. The high uptake observed with the use of both of the strategie is a de facto surrogate of not only program feasibility, but community acceptability as well ${ }^{8}$, and high levels of school enrolment of girls around 10 years of age was noted to be as high as $80 \%$, nationally. ${ }^{15}$

Other studies of HPV vaccine uptake through schoolbased strategies, such as those in Australia, Scotland, and Spain, ${ }^{16,17}$ have found similar levels of uptake. Recent analysis of programs operating in low-resource settings like Uganda, such as those in Rwanda, Tanzania, and other developing countries, demonstrated uptake ranging from 77 to $93 \%$, depending upon the delivery model used. ${ }^{18,19,20}$

Our study found factors that facilitated successful delivery of HPV vaccine included: coordination between health and education officials for implementation in schools (as done through the micro-planning exercise in this program); designing delivery strategies based on a good understanding of the current system and opportunities for synergy (as was identified by prior formative research); teacher involvement when vaccinating at schools; and implementation through the regular EPI system, structure, and human resources, with visible government endorsement and ownership of the program.

Binagwaho and colleagues have noted the critical role of delivery through the routine immunization program for HPV vaccine introduction in Rwanda, ${ }^{18}$ and Watson-Jones and colleagues emphasized that one of the limitations of their trial was that it was not closely linked with standard mechanisms of vaccine delivery in Tanzania. ${ }^{19}$

School-based delivery of HPV vaccines has special challenges, even in highly resourced places such as Australia. Cooper-Robbins, et al. found the logistics of coordinating with the school particularly difficult. ${ }^{21}$ Simiar constraints related to logistics were also found in this study in Uganda.
Of special note was the challenge of determining eligibility in the age-based delivery strategy. One consequence of this was the vaccination of girls outside the target age group, leading to an overestimation of coverage for the age-based strategy, noted as $105 \%$ in the administrative records. A population-based representative sample of households with girls who were 10 years of age at the time of the program administration in Nakasongola was conducted to measure coverage achieved by the delivery strategy and found only $52 \%$ of 10 -yearolds in the first year were fully vaccinated. ${ }^{8}$ In light of these findings of the challenges of age-based eligibility in Uganda, selecting girls for HPV their orade in school may be easier to inplenent, bu it would be important to ensure that all vaccine doses are delivered during the same calendar year to minimize loss to follow-up that may arise due to pupils changing schools in the subsequent calendar year. Grade-based selection for immunization programs is not for certai antigens, such as HPV, hepatitis B, tetanus, and acelluar pertussis, ${ }^{22}$ and in the HPV vaccination program in Scotland, primary grade five adolescent girls are targeted. ${ }^{23}$

As highlighted, loss to follow-up was high where girl This could be attributed to that fact that eligible girls were scattered across multiple classes ${ }^{8}$ and identifying them across the entire school required enormous efforts and time by the health workers, and yet the health workforce in Uganda is limited. ${ }^{12,13}$ In addition, the school system in Uganda is designed in such a way that pupils in primary grades 1 and 2 study half days, yet health workers work for a full day; therefore with many schools to cover in a given day, it may be probable that health workers arrive at a school after the primary grades 1 and 2 pupils have already left school.

Preparing the health and education systems in terms of cold chain and resource allocation was a key component for the HPV vaccine demonstration project in Uganda, as this helped identify gaps in advance and defined training needs for key personnel. These attributes are helpful in successful program implementation and improvements, as they are key functionalities of the systems and essential for service delivery. ${ }^{24}$ Biellik, et al. found that an assessment of the health infrastructure in Uganda provided an opportunity for strategic planning and suggestions for improvements to support vaccine delivery ${ }^{13}$ were selected by age compared to selection by class.

\section{Conclusion}

Despite the challenges, the Government of Uganda, working through UNEPI, PATH, the districts of Ibanda and Nakasongola, and their partners, have demonstrated that delivery of HPV vaccine is feasible through use of existing health and education systems. It is evidently clear that selection, vaccination, and follow-up of eligible girls based on class/grade is much more feasible compared to selection by age; this was mainly due to the fact that most pupils, their teachers, and sometime parents do not know their right age. It is important to acknowledge the major role teachers play in the tion requires close collaboration between the ministries of education and health. The disruption to schools and health facilities due to HPV vaccination cannot be ignored, although good preparation and planning minimizes them, as the benefits of delivering HPV vaccine to young adolescent girls surpasses the disruptions.

HPV vaccine continues to be delivered in both districts that participated in this demonstration project and in twelve additional districts starting in $2012 . .^{25}$ The experience of Uganda has generated evidence for government decision-making related to HPV vaccine introduction and informed valuable lessons that are relevant and beneficial for other low-resource countries as they plan and prepare to introduce HPV vaccines as a primary prevention tool for cervical cancer.

\section{Conflict of Interes}

The authors declare they have no conflicts of interest.

\section{Acknowledgments}

We thank the following people who were critical to this project's success: Prof. Anthony Mbonye and Dr. Issa Makumbi (Uganda Ministry of Health), Dr. Possy Mugyenyi and Dr. Rachel Seruyange (Uganda National Expanded Programme on Immunization), Drs. Julius Bamwine and Louis Kaboine (Ibanda district), Drs. Gerald Ssekitto and Constance Kidaga (Nakasongola district), as well as all study participants. This study was supported with a vaccine donation from GlaxoSmithKline Biologicals SA. GSK was not involved in the demonstration project design, implementation, or evaluation; however, GSK was provided the opportunity to review a preliminary version of the publication for factual accuracy, but the authors are solely responsible for 
the final content and interpretation. This project was Bulletin of the World Health Organization. 2013; 91(8), funded in whole by a grant from the Bill \& Melind Gates Foundation. The views expressed herein are solely those of the authors.

\section{References} $585-592$.

12. Katahoire RA, Jitta J, Kivumbi G, Murokora D, Arube Wani J, Siu G, et al. An Assessment of the Readiness for Introduction of the HPV Vaccine in Uganda. The African Journal of Reproductive Health 2009; 12 (3): 159-172.

1. GLOBOCAN 2008, International Agency for Re- 13. Biellik R, Levin C, Mugisha E, LaMontagne DS, search on Cancer (http://globocan.iarc.fr/factsheets/ Bingham A, Kaipilyawar S, et al. Health systems and cancers/cervix.asp )

immunization financing for human papillomavirus vaccine introduction in low-resource settings. Vaccine cancer website. Available at: http://www.who.int/vac- 2009; 27 (44): 6203-6209.

cine_research/diseases/hpv/en. Accessed February 21， 14. MacQueen KM, McLellan-Lemal E, Barthalow K, 2010.

Milstein B. Team-based codebook development: structure, process, and agreement. In: Guest G, MacQueen $\mathrm{KM}$, editors. Handbook for team-based qualitative research. Lanham: Altamira; 2008. 119-136.

15. Uganda Bureau of Statistics. Demographic and Health survey 2011: preliminary results. Kampala, Uganda: Uganda Bureau of Statistics; 2012. Available at: http://www.measuredhs.com/publications/publication-pr18-preliminary-reports.cfm

16. Reeve C, De La Rue S, Pashen D, Culpan M, Cheffins T. School-based vaccinations delivered by general practice in rural north Queensland: an evaluation of a new human papilloma virus vaccination program. Commun Dis Intell 2008; 32 (1): 94-98.

17. Bonani P, Levi M, Latham NB, Bechini A, Tiscione E, Lai P, et al. An overview on the implementation of HPV vaccination in Europe. Hum Vaccin 2011; 7 (Suppl 1): $1-8$.

18. Binagwaho A, Wagner CM, Gatera M, Karema C, Nutt CT, Ngabo F. Achieving high coverage in Rwanda's national human papillomavirus vaccination programme. Bull World Health Organ 2012 August 1; 90 (8): 623-628

19. Watson-Jones D, Baisley K, Ponsiano R, Lemme F Remes P, Ross D, et al. Human papillomavirus vaccination in Tanzanian schoolgirls: cluster-randomized tria comparing 2 vaccine-delivery strategies. I Infect Dis 2012 September 1; 206 (5): 678-686

20. Ladner J, Besson MH, Hampshire R, Tapert L Chirenje M, Saba J. Assessment of eight HPV vaccination programs implemented in lowest income countries. BMC Public Health 2012; 12: 370

21. Robbins SC, Bernard D, McCaffery K, Skinner SR 'It's a logistical nightmare!' Recommendations for optimising human papillomavirus school-based vaccination experience. Sex Health 2010 September; 7 (3): 271-278. organisation \& policy. 2010. Available at: http://www. 22. Delage G. Routine immunization schedule for $\mathrm{Ca}-$ nadian children. Paediatr Child Health. 1999; 4 (1): 14 23. Crosbie EJ, Brabin L. Cervical cancer: problem solved? Vaccinating girls against human papillomavirus. BJOG. 2010; 117 (2): 137-142.

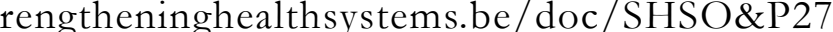
HS\%20ANALYSIS_FINAL.pdf

cination [press release]. Kampala, Uganda of Health (Uganda); September 4, 2012. Avallatr

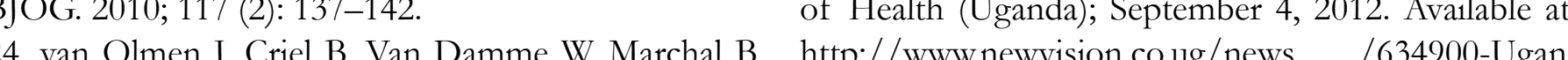
tems to make them stronger, studies in health services $\mathrm{html}$ 